\title{
Are you Searching for Ways to Find Information?
}

\author{
Colin Bird' \\ IBM $^{\text {ii United Kingdom Limited }}$ \\ Hursley Park \\ Winchester SO21 2JN \\ +44-(0)1962-816025 \\ colinl_bird@uk.ibm.com
}

\begin{abstract}
Effective information retrieval is important not only for its own sake but also for improving our understanding and allowing us to evolve our information into knowledge and wisdom, and thus to stimulate our creativity and enhance our culture. Classification enables us to discover those items that are about the subjects in which we are interested, but for greater effectiveness we must harness the power of communitydeveloped folksonomies in alliance with formal taxonomies. This paper discusses experiments aimed at realizing such an alliance.
\end{abstract}

\section{Categories and Subject Descriptors}

H.5.3 [Group and Organization Interfaces]

\section{General Terms}

Documentation, Design, Experimentation, Human Factors

\section{Keywords}

Information retrieval, classification, taxonomy, folksonomy, usability.

\section{INTRODUCTION}

"Astronomers say the universe is finite, which is a comforting thought for those people who can't remember where they leave things." (Woody Allen)

Woody Allen is not alone in the universe. We all have trouble recalling where we put things. Increasingly in the $21^{\text {st }}$ century, the 'things' that we lose track of are items of information, and access to information is increasingly what we need.

We also have a wide variety of strategies for overcoming the difficulties that occur with retrieving information. Some of us have highly-organised filing systems; some rely on more ad hoc approaches. We might ask whether a colleague with wellstructured system of storage is necessarily more effective at information retrieval than another with a "messy desk" who frequently seems to know exactly where in a certain pile to look. Who is to say that one scheme of mental metadata is any better than another?

The underlying message of this paper is that information is fundamental to Culture, Creativity, and Interaction: the theme of this conference.
I will begin by discussing a conceptual model of information and relate that model to the conference theme. I will then consider the potential influence that information classification might have on that model. Using examples from IBM online information centers, I will illustrate the role that classification, both formal and as the result of social tagging, might have in providing user assistance. The classification of online information presents both challenges and opportunities, which I shall relate back to the broader context and ultimately to the conference theme.

\section{CONCEPTUAL MODEL}

Information architecture is akin to designing buildings in which people will live or work, or do both, but the spaces that information architects (IAs) design are interactive and online. Exploring the nature of information architecture is outside the scope of this paper, but certain aspects are fundamental to retrieval. IAs must understand not only their product or service but also the use model: what questions will users be posing and how will those users interact with the design?

IAs organize the user assistance content for their product or service according to an information model, which describes the information deliverables, taking into account the way in which users will interact with that content.

The conceptual model that this paper considers is not specific to any product or service. This model is generic in that it describes the evolution of understanding from data through information to knowledge and then wisdom.

Consider the following extract from The Rock, by T S Eliot, published in 1934

The endless cycle of idea and action,

Endless invention, endless experiment,

All our knowledge brings us nearer to our ignorance

Where is the wisdom we have lost in knowledge?

Where is the knowledge we have lost in information?

T. S. Eliot alludes to dependency relationships between wisdom and knowledge, and between knowledge and information. However, Russell Ackoff [1] was probably the first to suggest a hierarchical relationship with wisdom at the top and data as the foundation. Awad \& Ghaziri [2] contributed to the evolution of the DIKW (Data, Information, Knowledge, Wisdom) model with a pyramid representation, which is the foundation for Figure 1.

Bellinger, Castro, and Mills [3] went on to suggest that the transformations could be related to levels of understanding, although they labelled their vertical axis as connectedness.

The DIKW model can also be related to retrieval, so the representation of the DIKW model in Figure 1 includes a second vertical axis, which indicates how our mental 
mechanisms might change according to the level at which we are retrieving.

\section{The DIKW model}

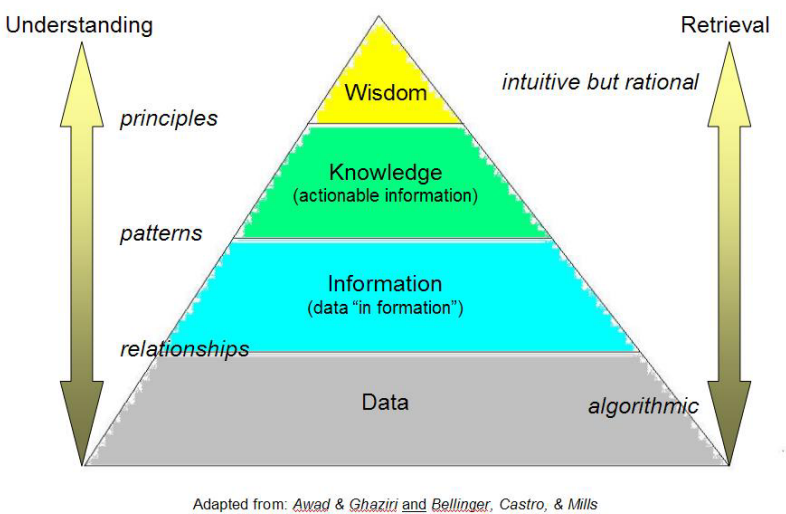

Figure 1. The DIKW (Data, Information, Knowledge, Wisdom) model shows how the human mind can move raw data up to higher planes by progressive organisation.

Relationships between data elements enable bits and bytes to gain meaning and thus become informative to us. As we move up the hierarchy, looking for patterns and deploying principles, we impose structure and organization, often by classifying or categorizing the information and knowledge.

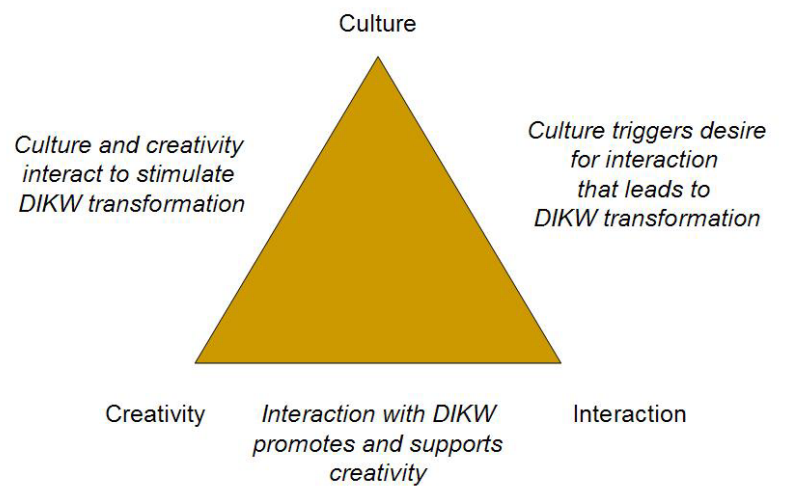

Figure 2. The conference theme of

Culture, Creativity, Interaction relates readily to the transformations inherent in the DIKW model.

Looking at the triangle, the interplay between the three concepts at the vertices is intuitive. Creativity rarely if ever occurs in isolation; most commonly it results from interaction with a stimulus, a process that necessarily involves information in one form or another. Culture is almost inevitably associated with knowledge and often with wisdom. Such associations are relatively easy to articulate but tend to minimise the importance of being able readily to access the information and the knowledge.

Arguably, the problems of information retrieval have always been with us, and typically our response has been to impose some form of organisation to facilitate access when needed. In recent years, we have certainly become a great deal more aware of the issues of information retrieval, and have turned increasingly to classification as one way to enable efficient and effective discovery.

Scientists and philosophers have used classification to capture their understanding of the world and to evolve increasingly sophisticated characterizations of the phenomena that they observe. Information specialists have classified their resources to make information easier to find.

Classification is important to both understanding and retrieval, and to moving up the DIKW pyramid.

\section{INFORMATION CLASSIFICATION 3.1 Classification in context}

We create structures to organise our information and to support our understanding, applying techniques that range from simple labelling, through indexing, to classification using formally controlled vocabularies.

However, these techniques do require additional effort and are not infallible. Search remains the most commonly-used approach for information retrieval, but even with progressive refinement, free-text searching is still a rather blunt tool. The hits contain the search terms, but are not necessarily about them. Moreover, the selection of the hits that we see is likely to be influenced by web site owners who know how to achieve high ranking for their pages.

A desirable goal is to narrow the search space, by having the system select only the items that are about the subjects that a user is interested in. Tagging items of information with classification metadata enables users to specify those subjects.

Filtering offers an alternative approach, but one with a different objective: filtered output has been processed to omit content that is not relevant, whereas tagged output has been processed to identify the relevance of the content.

The two approaches can be used together, by looking for items with specified tags within an already-filtered information space.

\subsection{Taxonomies and folksonomies}

The term taxonomy has been familiar to biological scientists since the 18th century, when Carl Linnaeus developed the system of classification that became known as the Linnaean taxonomy. In the information systems context, we can define a taxonomy as: "A classification of concepts into a hierarchical structure according to whether the concepts are more general or more specific."

The familiar form of the folksonomy is the tag cloud, in which the most-used tags grow larger at the expense of less popular terms. Folksonomies provide a community form of relevance feedback, in which end-users attach descriptive tags, or labels, to items of information and can make use of the descriptive labels provided by other members of the community.

The term folksonomy is often used as a synonym for social tagging, although the latter term actually describes a process, one outcome of which is the vocabulary emanating from the tagging activity. The folksonomy is the vocabulary space.

\section{COLLABORATIVE TAGgING}

The Wikipedia article about folksonomy and social tagging[4] prefers to emphasize the collaborative nature of the exercise, and that is a notion on which this paper seeks to build. However, we shall be concerned with the role of collaborative tagging and folksonomies in knowledge organisation and discovery, rather than with the social aspect.

The same Wikipedia article also explores not only the merits of the folksonomy approach but also some of the challenges, including the compromise with taxonomies.

The IBM Corporate Information Office makes the following distinction between the two approaches to information tagging: 
"A folksonomy is a collection of terms which are used by a community to categorize data. Unlike systems which have an official taxonomy with formal controlled keywords in a hierarchy, a folksonomy is a flat namespace built by the end-users choosing words which have meaning to them."

This distinction naturally raises the question of whether folksonomies and taxonomies rival or complement each other.

Both provide terms that inform users what an item of information is_about and both seek to enhance retrievability:

- Taxonomies aim to improve information access by providing a consistent and unambiguous structure. Each term should have a definition.

- Folksonomies have little or no concern with consistency but aim to make it easier for users to tag items of information and to access other users' tags.

Both use term lists, albeit with different styles of organisation. Initially, folksonomic vocabularies are uncontrolled although, over time, the participating community inevitably exerts an elective form of control.

Some practitioners see the difference more starkly, because they are concerned about our ability to cope with the rapidly expanding amount of information available and the corresponding exponential decline in the half-life of knowledge (how long it takes for half of it to become obsolete). They urge us to shift our information processing from content to connections. Their view can be summarized as follows:

- Classification imposes structure (and so freezes the content)

- Organising in a community context encourages enduser input.

Although this binary stance is understandable, it is also regrettable: we really need the two 'onomy's to complement each other, and we should be looking towards an alliance.

\subsection{Towards an alliance}

We have already begun to see end-user participation in vocabulary evolution, and if we can find ways to manage the relationship between folksonomies and taxonomies, which are more formally controlled, we can expect to see the emergence of dynamic vocabularies. Being dynamic does not imply anarchy, rather that we will have more flexible regimes of control.

Within IBM we are developing the classification of topics in information centers, which are Eclipse-based and provide IBM's primary source of online information about its products and solutions.

We are currently developing a classification scheme that involves a base taxonomy and extension taxonomies, with a governance process to control them and the terms within them.
We aim to tell our customers what the topics in our online information centers are about, particularly by controlling our vocabularies.

There is, however, a trade-off. Retrieval using a controlled vocabulary will have high precision, but might have low recall, failing to retrieve some documents that are actually relevant, especially if a vital tag is missing. To address the potential difficulties with formal taxonomy-based schemes, we plan to experiment with interfaces that facilitate information discovery with a combination of folksonomy tags and classification metadata.

\subsection{Dynamic vocabulary experiments}

The basis of the experiments will be an IBM information center that includes content classified according to a formal taxonomy, with function added to enable collaborative tagging.

The issues that we intend to explore include, but are not confined to: usability, in a wide sense of the term; scalability, particularly if the number of terms in the folksonomy exceeds significantly the number of subjects in the taxonomy; and the development of an appropriate life-cycle model for folksonomy-type metadata.

Furthermore, governance processes will be crucial to the success of dynamic vocabularies and particularly to schemes that involve hierarchies of general and specific scheme components.

\section{CONCLUSIONS}

Dynamic vocabulary schemes that harness the merits of both formal taxonomies and community-developed folksonomies clearly offer great opportunities for improving both retrieval and understanding. Such schemes also present challenges, especially with regard to usability, scalability, and the evolution of effective governance processes, but we believe these challenges can be met. The reward will be to facilitate progress up the DIKW pyramid.

Information retrieval is very much dependent on effective interaction, and improving our access to those items that are about the subjects in which we are interested must help to stimulate both creativity and culture.

\section{REFERENCES}

[1] Ackoff, R. L. From Data to Wisdom. Journal of Applied Systems Analysis, 16, 1989, 3-9

[2] Awad, E. M. and Ghaziri, H. M. Knowledge Management. Upper Saddle City, NJ, Pearson Educational International, 2004

[3] Bellinger, G., Castro, D., et al. Data, Information, Knowledge and Wisdom. http://www.systems-thinking.org/dikw/dikw.htm

[4] Wikipedia, Folksonomy, http://en.wikipedia.org/wiki/Social_tagging

\footnotetext{
${ }^{\mathrm{i}}$ Any views expressed in this paper are entirely those of the author and do not represent those of the IBM Corporation.

${ }^{\text {ii }}$ IBM is a trademark of IBM Corporation in the United States and other countries.
} 